\title{
Color Segmentation for Sixth Sense Device
}

\author{
Md. Shoaibuddin Madni and Ravindra N. Rathod
}

\begin{abstract}
Sixth sense device is a gesture recognition device which uses hand gestures for recognition and performing various operations. Color segmentation is an important task for sixth sense device after color recognition. A RGB color space is an additive color space based on RGB color model. This proposed color model is used to recognize and segment various colors from real time images. A RGB color space model consists of three chromaticity's i.e. Red, Green \& Blue additive primaries, and can produce any chromaticity that is defined by those colors [1]. The continuous frames of video are converted to RGB color space, where the color information is extracted for color recognition. Here we capture video through HD webcam. After color recognition, the color is being segmented. The color segmentation involves RGB color normalization, centroid calculation. The Euclidean distance formula is being used for color recognition \& centroid location. The coding for all the above steps is being done using MATLAB 7.1 version.
\end{abstract}

KeyTerms--- Human Computer Interface, Sixth Sense device, Gesture Recognition , Color Recognition , Color Segmentation, MATLAB , Image Processing

\section{INTRODUCTION}

We've evolved over millions of years to sense the world around us. When we encounter something, someone or some place, we use our five natural senses which include eye, ear, nose, tongue mind and body to perceive information about it. This information helps us make decisions and chose the right actions to take. But arguably the most useful information that can help us make the right decision is not naturally perceivable with our five senses, namely the data, information and knowledge that mankind has accumulated about everything and which is increasingly all available online.

"Sixth Sense Technology", it is the newest jargon that has proclaimed its presence in the technical arena. This technology has emerged, which has its relation to the power of these six senses. Our ordinary computers will soon be able to sense the different feelings accumulated in the surroundings and it is all a gift of the "Sixth Sense Technology" newly introduced. Sixth sense technology is a revolutionary way that augments physical world directly without using dedicated electronic chips. Sixth sense is a wearable gestural devicewhich acts as a gestural interface and enlarges the physical world around us with digital information and help the user to use natural hand

Md. Shoaibuddin Madni, Research Scholar, Dept. of Electronics \& Communication Engineering, SDM College of Engineering \& Technology, Karnataka, India.

Ravindra N Rathod, Assistant Professor, Department of Electronics \& Tele-Communication Engineering, Government College of Engineering, Maharastra, India.

DOI:10.9756/BIJRCE.8208 gestures to interact with the digital information through it. This technology is becoming most popular popularbecause of the fact that any task or operation can be performed with the gestures only.

Nowadays, the majority of the human-computer interaction(HCI) is based on the electromechanical devices such as keyboards,mice, joysticks, electronic pens, touch screens,gamepads and so on. Compared with all other HClinterfaces, devices that could recognize body position, handgestures, sound and speech, facial expression and other aspects of human behavior are more intuitive andpowerful.

This technology is gaining its popularity for its usability, simplicity and ability to work independently.

The camera tracks a pointer / color cap which makes gesture. Hence color segmentation plays a vital role for sixth sense device. The segmented real time images consists of each color segmented pixel values and appropriate values between other colors.

Real time images are segmented by using RGB Color model. These values of ' $R$ ', ' $G$ ' \& ' $B$ ' vary for different colors. These values are noticed for different colors and programmed in MATLAB and are used for segmenting the various colors.

\section{Motivation}

A gesture recognition device provides advanced dynamic environments where humans can make assorted types of interface for interaction with media and information without physical restrictions. Computers are a fundamental part of any educational core curriculum hence the educational systems also provide a very large domain of applications for gesture recognition. The user develops the interface which uses common mathematical tool i.e. MATLAB for this Gesture recognition device. Evolution of computer, tablets, desktops to palmtops has made the presence of human computer interaction felt in nearly all the aspects of life. The key board, mouse etc. lack sensitivity in this gesture recognition device. Hence a lot of research needs to be done for Human Computer Interface.

\section{PROBLEM STATEMENT}

Various applications and algorithms are designed for color segmentation. Camera is used for capturing images of set of color caps. Numerous applications related to color recognition have been designed for presenting, pointing, virtual workbenches, VR etc. The input to the device is video from webcam or camera. Video consists of number of frames or objects. All frames are in RGB format. These are being converted to LAB format to get color coefficients. These recognized colors are segmented using several algorithms. One 
of the major challenges in this project is complexity and accuracy of a result.

\section{RELATED WORK}

Sixth Sense Device developed as a wearable gestural interface. This is developed byMaes' MIT group, which includes seven graduate students, working on the gesture recognition device. i.e. the device helps user in recognizing gestures and performing various relevant operations without using actual device. They initially produced a wristband that would read a RFID tag to know, for example, to know the details of textbook a user is holding in a store [5].

Color Recognition is the preliminary step for gesture recognition. The video from the camera or webcam is converted into number of frames. The frames are further converted from RGB to Lab or any other color format. These formats give the pure Color Components by removing or reducing the luminosity. Calibration process captures images and extracts various colors and saves it. Euclidean distance formula is used to compute every pixel value, based on pixel values various colors are being recognized [6].

Theproposed bare hand gesture recognition system composedof image pre-processing, skin segmentation, feature extractionand gesture classification.The image pre-processing module transforms the raw imageinto the desirable feature vector which mainly includesconverting the color images into the hue images and reducingnoise. Theskin segmentation module localizes and segmentsthe skin region from the whole image and eliminates theforearm region with the help of a wrist line. The featureextraction module extracts a set of distinct parameters torepresent each gesture and distinguish the different gestures [3]

Hand gesture segmentation is the key and major premise tothe analysis and identification. Quality of the gesturesegmentation directly affects the rate of recognition. Effectiveuse of various information such as color,motion and geometricinformation is the key of the study. Hand gestures in acomplex background are very difficult to separate, no maturetheory exist as a guide [2].

\section{OBJECTIVE}

The main objective of the paper can be divided into the following points.

1. To segment hand from real time images by using background subtraction method.

2. To extract the features from the color caps.

3. Perform relevant operations as per commands given by user.

4. To write a MATLAB code for all of the above steps.

\section{METHODOLOGY}

Here we describe about the working of sixth sense technology. Sixth sense device uses a gestural interface and uses hand gestures for communication. Sixth Sense device comprise of its components such as, Camera to record the gesture, Projector to display, Mobile component for computation etc. The preliminary step is to convert the video into images. As the image consists of the color components i.e. Red, Green \& Blue. Hence the images should be converted into the RGB Color image. This RGB image can be used to extract the color. Since the gestures are defined by using color caps, hence the color should be recognized \& segmented. After the color segmentation, the centroid for the image should be located. The distance between the centroids can be used to define the particular gesture. For ex. Distance between R, G = 20 to $25 \mathrm{~mm} \mathrm{\&} \mathrm{Y,} \mathrm{B=} 20$ to $25 \mathrm{~mm}$. The camera should capture the image. Likewise any number of gestures can be defined. The working methodology can divided into three parts.

1. Color Recognition.

2. Color Segmentation.

3. Actuation.

\section{Color Recognition}

The first step for Sixth Sense device is to recognize various colors. This Methodology uses a Euclidean Distance formula to compute the pixel values and recognize multi colors. The captured multi-color image is takenand using a Roipoly command in MATLAB we select the region of interest of an image. The selected portion pixel values are extracted and stored. The process converts the video into number of frames and each frame value is computed with the stored pixel values using Euclidean distance formula. Based on matching distance between various pixel values we recognizemulti-color.The color vision system is based on a color recognition technique that is insensitive to changes in viewing parameters such as viewing angle and distance. In this task of the color recognition system is to find person wearing a green, red, blue, yellow color caps.

\section{a) Calibration}

We first convert the input image from RGB to LAB format (LAB format separates the luminosity component and gives only color information. Then we prompt user to select sample region of each color and store the color information.

\section{b) Identification}

We take input image then convert to $\mathrm{LAB}$ format. Then we calculate the Euclidian distance of each pixel with stored samples. Based on the matching distance we classify every pixel in its respective class.

After calculating the Euclidean distance with the captured image we take the mean and median to get the average pixel values. Euclidean Distance is calculated by using formula

$$
\sqrt{ }(X 2-X 1)-(Y 2-Y 1)^{2}
$$

\section{c) Actuation}

The color shown on a webcam gets captured and compared with the stored pixel values. Based on the pixel values different colors will be recognized (ex Green, Yellow, Blue, Red.) and shown on a desktop window.

\section{Color Segmentation}

Color segmentation is the very first step in gesture recognition, hand tracking and recognition. We propose a newest technique. i.e. RGB normalization for color 
segmentation of color. This method captures pixel values of a various colors separately. Each color is normalized and stored. Based on matching distance calculated by Euclidean distance formula with the original pixel values and normalized pixel values we segment various colors. After segmentation we take each segmented color and calculate the centroid of each. Later distances between various centroid points of various colors are calculated.

\section{a) RGB Normalization}

Segmentation is always a very important and basic task while detecting a particular object. For example, in thispicture, User wearing color markers on his fingers to track the motion of fingers and do some task according to movement of fingers. But detecting an object is not an easy task. Light also play crucial role. For example, if you have unidirectional source of light then it creates shadow and different shades of colors on object.

So to reduce the effects of light, Normalization of color space is helpful. Normalization removes highlighted regions, shadows and makes that object easier to detect.

Now some mathematical work starts here, following equation convert a pixel to normalized pixel.

$$
\begin{aligned}
& \mathrm{f}(\mathrm{x}, \mathrm{y})=(\mathrm{R}, \mathrm{G}, \mathrm{B}) \\
& \text { Total }=\mathrm{R}+\mathrm{G}+\mathrm{B} \\
& \mathrm{R}^{1}=(\mathrm{R} / \text { Total }) * 255 \\
& \mathrm{G}^{1}=(\mathrm{G} / \text { Total }) * 255 \\
& \mathrm{~B}^{1}=(\mathrm{B} / \text { Total }) * 255 \\
& \text { Thus } \mathrm{g}(\mathrm{x}, \mathrm{y})=\left(\mathrm{R}^{1,} \mathrm{G}^{1,} \mathrm{~B}^{1}\right)
\end{aligned}
$$

Let $\mathrm{R}, \mathrm{G}, \mathrm{B}$ are pixel values, to normalized the pixel, divide the individual color component with 'total' and multiply by 255. Here 255 is scaling factor. You can use any other scaling factor. As this image is 8 bit scaling value is 255 .

\section{b) Centroid Calculation}

After RGB normalization the centroid of each color is calculated. The distance between centroids of each color with other color component is calculated using the Euclidean Distance Formula.

$$
\sqrt{(X 2-X 1)-(Y 2-Y 1)^{2}}
$$

\section{PROPOSED AlGORITHM}

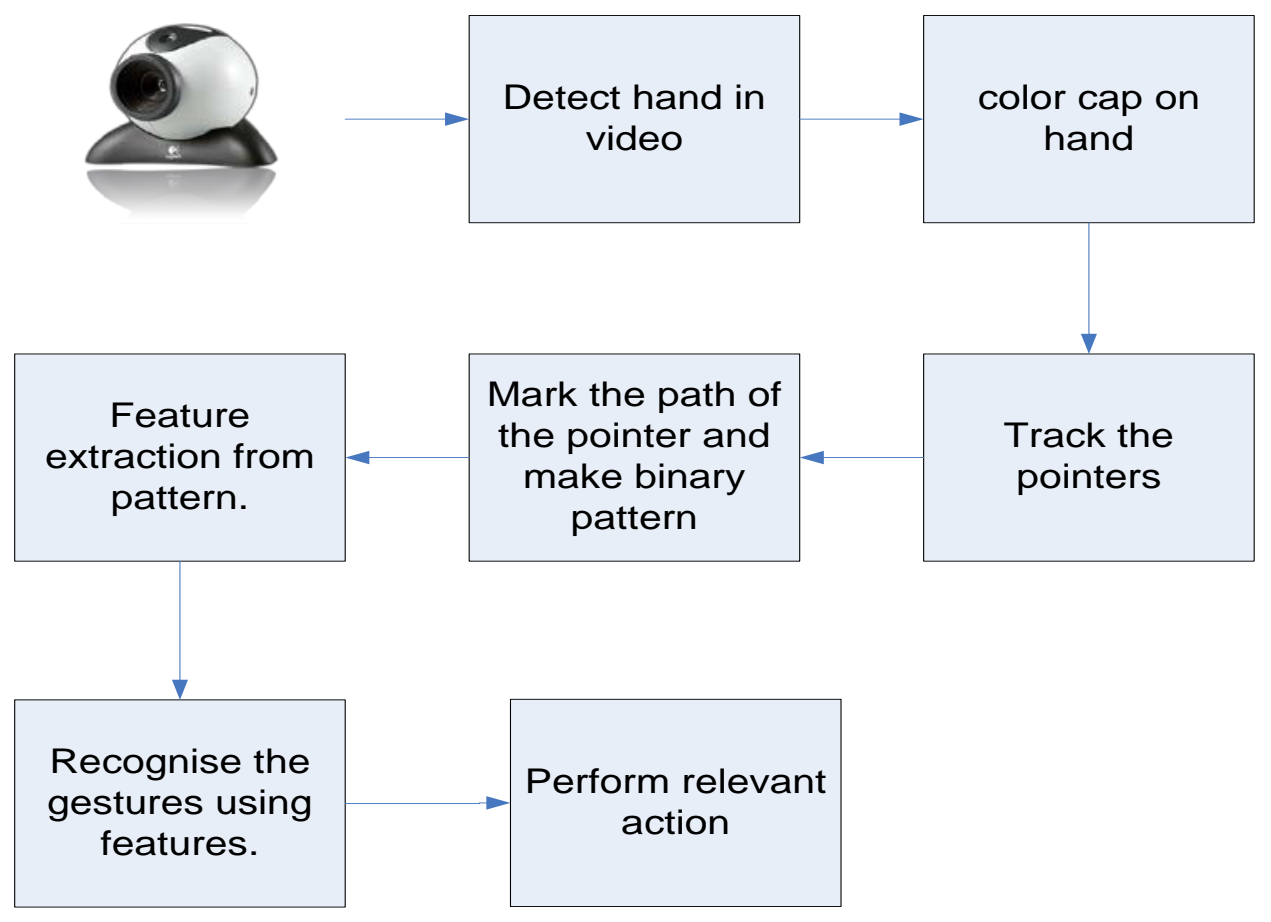

Figure 1: Proposed Algorithm

\section{IMPLEMENTATION RESULTS}

The image processing, color recognition and color segmentationis simulated in MATLAB to process the image from continuous frames i.e. video obtained from camera to segment required colors only. The image processing, color recognition and color was performed with the machine of Pentium Quad core processor at 2.2 GHz. Clock frequency, 3
GB RAM and standard onboard graphics unit of Intel Motherboard. The algorithm captures the sample image where the user wears colorcaps and store the color pixel information. The sample image and its output image after color recognition \& segmentation are shown below. 


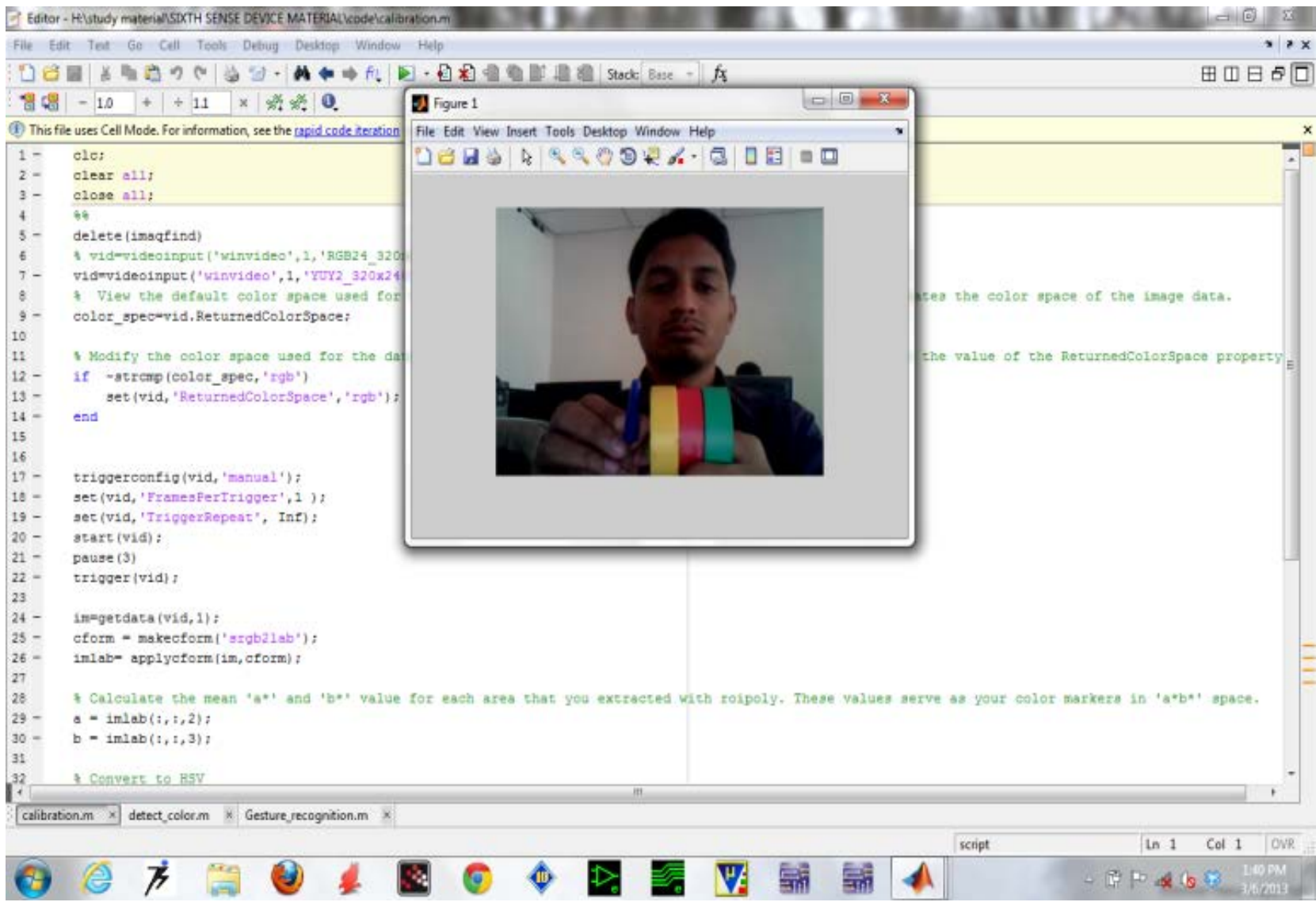

Figure 1.a: Calibration

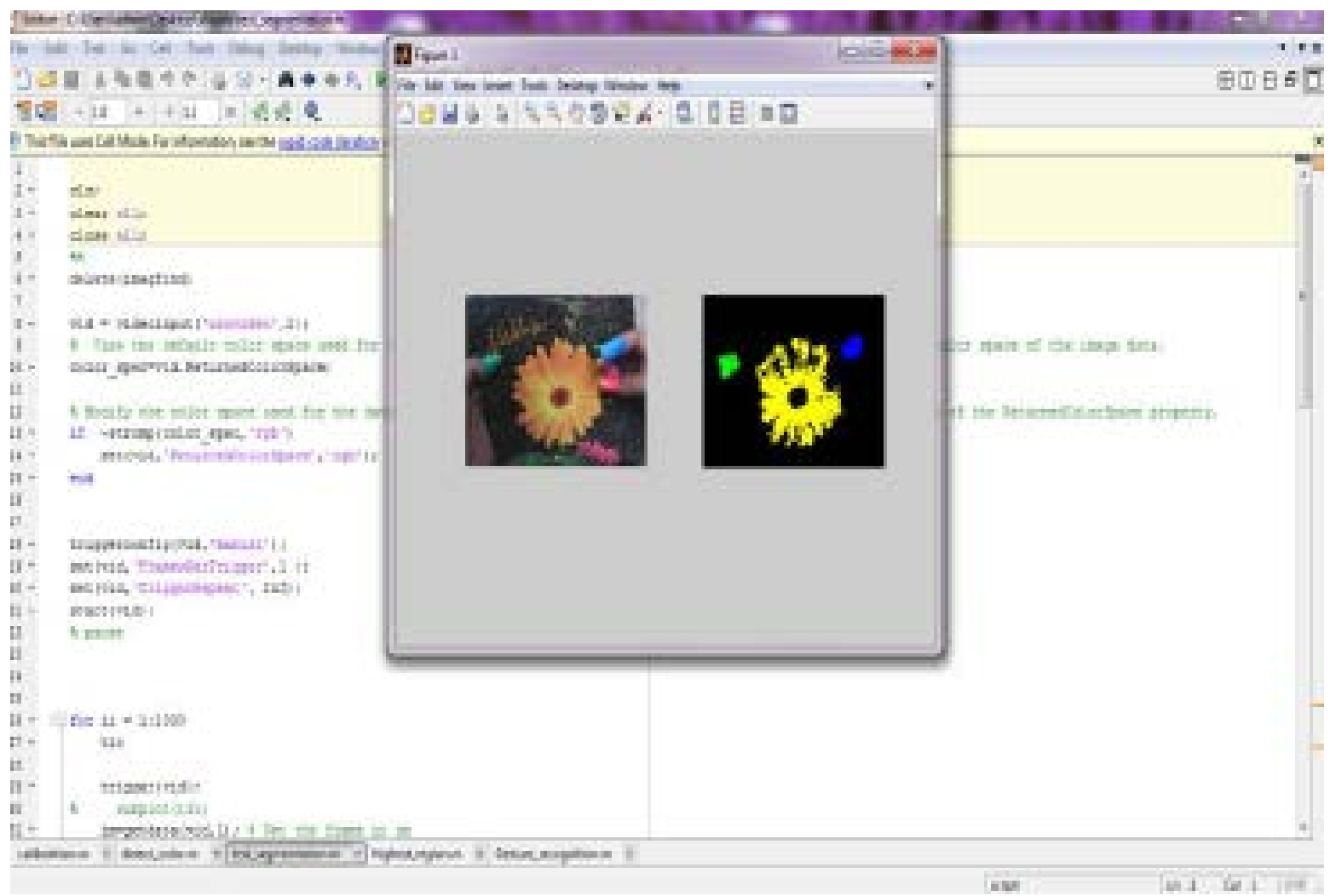

Figure1.b: Multi Color Segmentation 


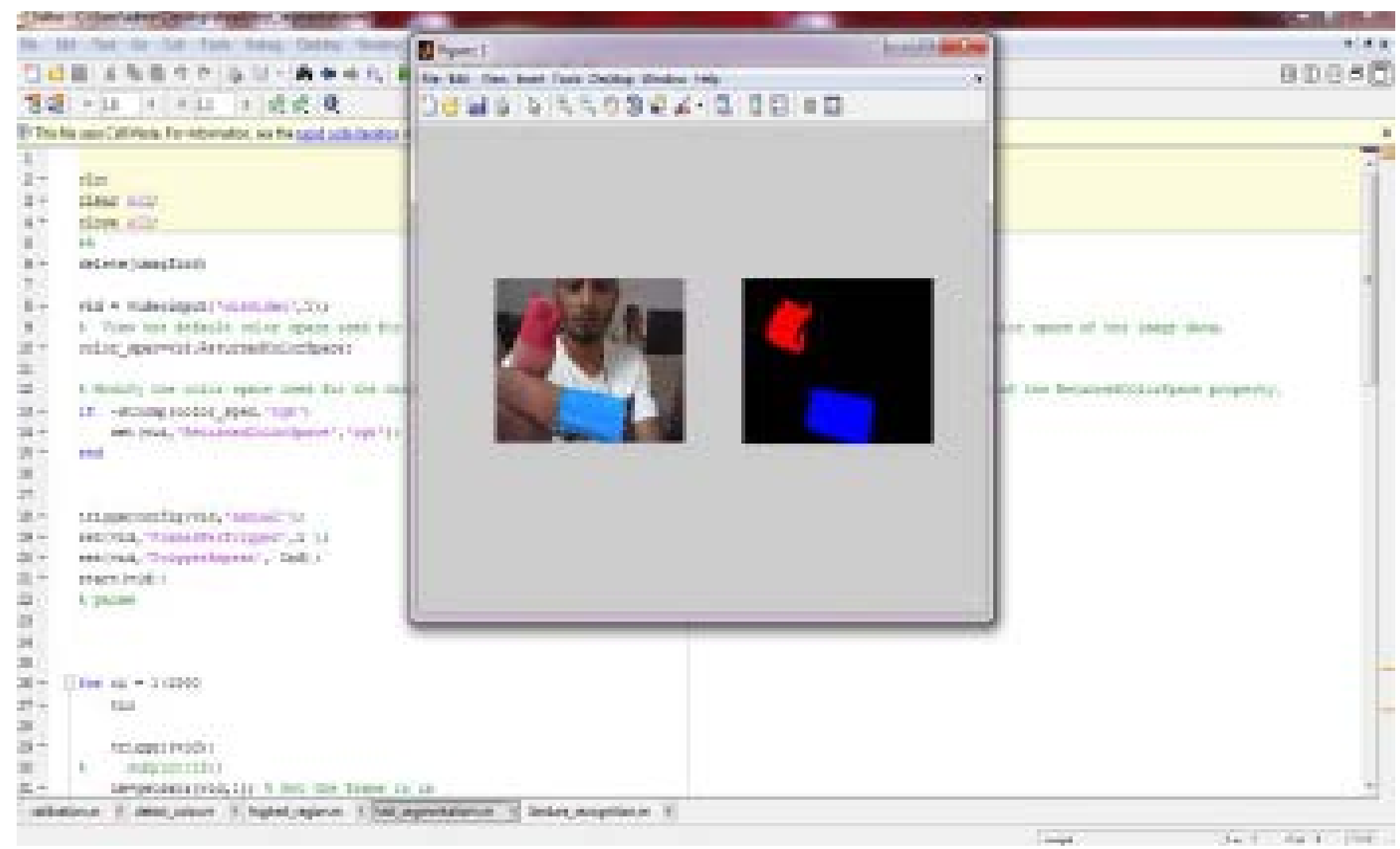

Figure 1.c: Multi Color Segmentation

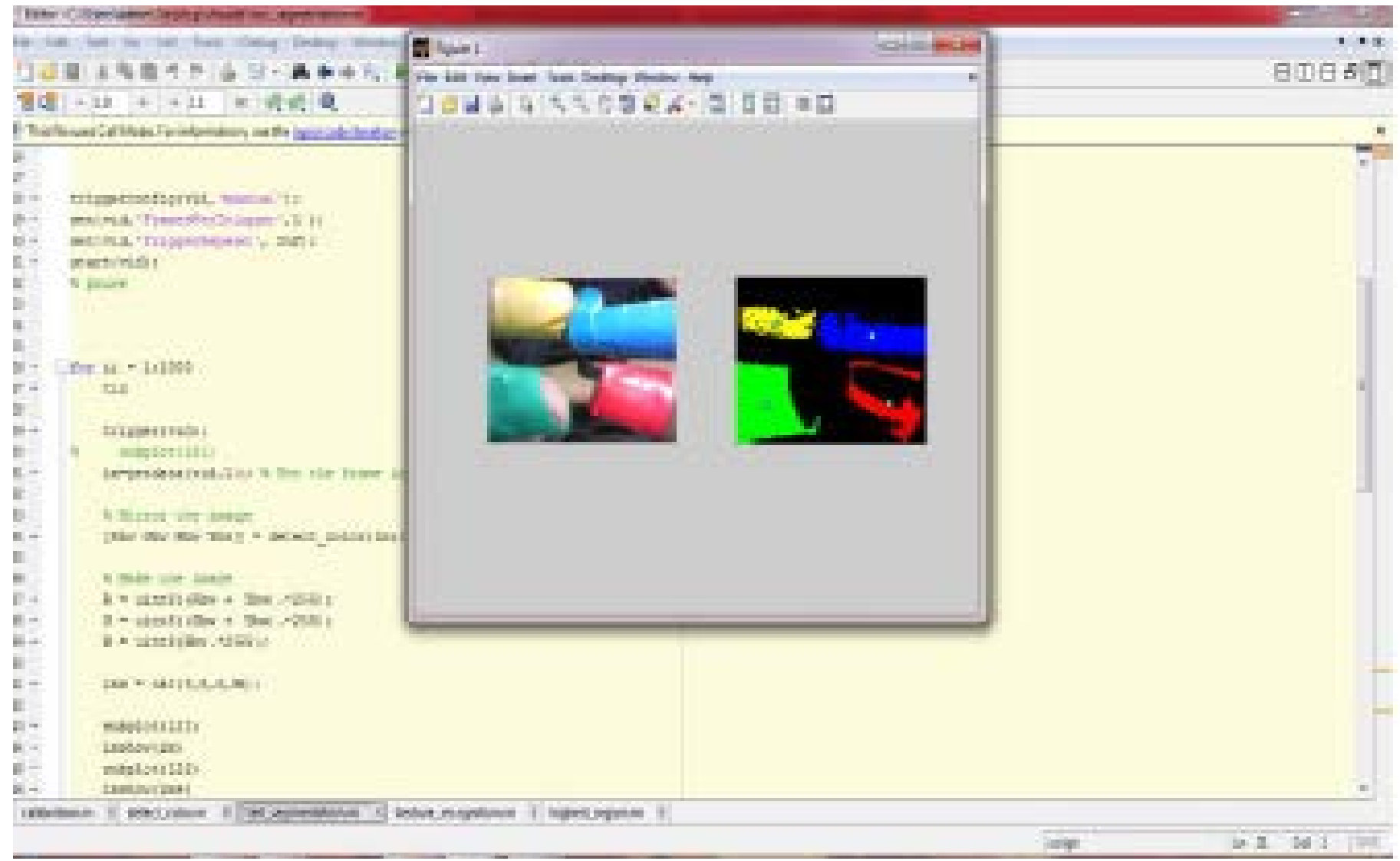

Figure 1.d: Centroid Location 


\section{CONCLUSION}

The use of Image processing in calibration, color recognition, color segmentation and actuation in MATLAB for the implementation of our proposed projecti.e.Color Segmentation for Sixthe Sense Device proved to be practically successful with minimal errors. The approach gets huge potential once it gets optimized to reduce the noise, the time and complexity is more, with the help of hardware having better specifications. This approach gets more power forfuture advanced applications which can change the mobile world also.

\section{FUTURE WORK}

Sixth Sense has the ability to remove the gap between the two worlds and observing the implementation of the system it is clear that the designed system has a huge potential to be used in immense and can be said that the proliferation of this technology may be there within this 2020 and sufficient awareness of this technology will lead to further development of any technology [1] [2].

The key here is that Sixth Sense recognizes the objects around you, displayinginformation automatically and letting you access it in any way you want, in the simplest waypossible. Device

The two main operations are proposed for Sixth Sense

1. Mouse control operation which can perform all operation of a mouse.

2. Image Modification i.e. Image rotation, Zoom In, Zoom out.

\section{REFERENCES}

[1] P. Agrawal and K. Gupta, "Mouse Movement through finger by image grabbing using sixth sense technology", IJESAT, Vol.2, No.2, 2012.

[2] B. Hong and Z. Xinggui, "Study on hand gesture segmentation", International Conference on Multimedia Technology, 2010.

[3] M.M. Hasan and P.K. Misra, "Gesture recognition using modified HSV segmentation", International Conference on Communication Systems and Network Technologies (CSNT), Pp.328-332, 2011.

[4] T. Gervers and A.W. Smeulders, "Color based object recognition", The journal of pattern recognition society, 1997.

[5] S. Jophin, M.S. Sheethal, P. Philip and T.M. Bhruguram, "Gesture Based Interface Using Motion and Image Comparison”, International Journal of Advanced Information Technology, Vol.2, No.3, Pp.37, 2012.

[6] Md. ShoaibuddinMadni and ReshmaNadaf, "Color Recognition for Sixth Sense Device”, National conference on Electronics, Communication \& Advance Networks, 2013.

[7] E.J. Holden and R. Owens, "Recognizing moving hand shapes," in Proceedings of International Conference on Image Analysis and Processing, Pp.14-19, 2003.

[8] C. Nolker and H. Ritter, "Visual recognition of continuous hand postures”, Neural Networks, Vol.13, No.4, Pp.983-994, 2002.

[9] R. Stiefelhagen, C. Fugen, P. Gieselmann, H. Holzapfel, K. Nickel and A. Waibel, "Natural Human-Robot Interaction using Speech, Gaze and Gestures”, Proceedings. 2004 IEEE/RSJ International Conference on Intelligent Robots and Systems, Pp. 2422-2427, 2004.

[10] S. Waldherr, R. Romero and S. Thrun, "A gesture based interface for human-robot interaction”, Autonomous Robots, Vol.9, Pp. 151-173, 2000.

[11] F.A. Bertsch and V.V. Hafner, "Real-time dynamic visual gesture recognition in human-robot interaction", IEEE-RAS International Conference on Humanoid Robots, Pp. 447-453, 2009.

[12] P. Viola and M.J. Jones, "Robust Real-Time Face Detection", International Journal of Computer Vision, Vol.57, No.2, Pp.137-154, 2004.
[13] M.J. Jones and J.M. Rehg, "Statistical color models with application to skin detection”, Tech. Rep. Tech. Rep. CRL 98/11, Cambridge Research Laboratory, Vol.46, No.1, Pp.81-96, 1998. 